\title{
BALANCED VALUATIONS AND FLOWS IN MULTIGRAPHS
}

\author{
F. JAEGER
}

\begin{abstract}
A balanced valuation of a multigraph $H$ is a mapping $w$ of its vertex-set $V(H)$ into $R$ such that $\forall S \subseteq V(H)$ the number of edges of $H$ with exactly one vertex in $S$ is greater than or equal to $\left|\Sigma_{v \in S^{w}}(v)\right|$; we apply the theory of flows in networks to obtain known and new results on balanced valuations such as:

A cubic multigraph has chromatic index 3 if and only if it has a balanced valuation with values in $\{-2,+2\}$ (Bondy [5]).

Every planar cubic 2-edge connected multigraph has a balanced valuation with values in $\{-5 / 3,+5 / 3\}$.

Every planar 5-regular 4-edge connected multigraph has a balanced valuation with values in $\{-3,+3\}$.
\end{abstract}

\section{Introduction.}

1. Basic definitions and notations. A multigraph $H$ (respectively: directed multigraph $G$ ) is a finite nonempty set $V(H)$ (respectively $V(G)$ ) of vertices together with a finite family $E(H)$ (respectively $A(G)$ ) of 2-element subsets of $V(H)$ called edges (respectively: of ordered pairs of elements of $V(G)$ called arcs).

When we replace each edge $e=\left\{v, v^{\prime}\right\}\left(v, v^{\prime} \in V(H)\right)$ of a multigraph $H$ by an arc $\left(v, v^{\prime}\right)$ or $\left(v^{\prime}, v\right)$ we obtain a directed multigraph $G$ (with $V(G)$ $=V(H))$ which will be called an orientation of $H$.

For a directed multigraph $G$, for any $S \subseteq V(G)$, let

$\omega_{G}^{+}(S)=\left\{a \in A(G) \mid a=\left(v, v^{\prime}\right), v \in S, v^{\prime} \notin S\right\}$.

$\omega_{G}^{-}(S)=\omega_{G}^{+}(V(G)-S)$.

$\omega_{G}(S)=\omega_{G}^{+}(S) \cup \omega_{G}^{-}(S)$.

For any vertex $v$ of $G$ :

The outdegree of $v$ in $G$ is $d_{G}^{+}(v)=\left|\omega_{G}^{+}(\{v\})\right|$.

The indegree of $v$ in $G$ is $d_{G}^{-}(v)=\left|\omega_{G}^{-}(\{v\})\right|$.

The degree of $v$ in $G$ is $d_{G}(v)=\left|\omega_{G}(\{v\})\right|=d_{G}^{+}(v)+d_{G}^{-}(v)$.

For a multigraph $H$, for any $S \subseteq V(H)$, let $\omega_{H}(S)=\{e \in E(H)|| e \cap S \mid=1\}$.

For any vertex $v$ of $H$, the degree of $v$ in $H$ is $d_{H}(v)=\left|\omega_{H}(\{v\})\right|$. Other definitions not given here will be found in [1] and [2].

2. Flows in directed multigraphs. Let $G$ be a directed multigraph with $|A(G)|=m \geqslant 1$ and $A(G)=\left\{a_{1}, \ldots, a_{m}\right\}$. A flow in $G$ with values $\phi_{i}$ $(i=1, \ldots, m)$ is a vector $\phi=\left(\phi_{1}, \ldots, \phi_{m}\right) \in Z^{m}$ such that

$$
\forall S \subseteq V(G) \quad \sum_{a_{i} \in \omega_{G}^{+}(S)} \phi_{i}-\sum_{a_{i} \in \omega_{\bar{G}}^{-}(S)} \phi_{i}=0 .
$$

Received by the editors June 15, 1975.

AMS (MOS) subject classifications (1970). Primary $05 \mathrm{C} 15$. 
The flows in $G$ form a submodule of $Z^{m}$. For every $p$ and $q$ in $Z$ let $[p, q]=\{z \in Z \mid p \leqslant z \leqslant q\}$. Let $b_{1}, \ldots, b_{m}, c_{1}, \ldots, c_{m}$ be elements of $Z$ with $b_{i} \leqslant c_{i} \forall i \in[1, m]$. Then we have [1]

Feasible Flow Theorem. There is a flow $\phi=\left(\phi_{1}, \ldots, \phi_{m}\right)$ in $G$ with $\phi_{i} \in\left[b_{i}, c_{i}\right] \forall i \in[1, m]$ if and only if

$$
\forall S \subseteq V(G) \quad \sum_{a_{i} \in \omega_{G}^{+}(S)} c_{i}-\sum_{a_{i} \in \omega_{\bar{G}}^{-}(S)} b_{i} \geqslant 0 .
$$

3. Flows and face colorings for planar multigraphs.

Proposition 1. A planar directed multigraph $G$ is face colorable with $k$ colors $(k \geqslant 2)$ if and only if there is a flow in $G$ with values in

$$
[-k+1,-1] \cup[1, k-1] \text {. }
$$

REMARK. The two preceding equivalent properties imply that $G$ has no acyclic arc (bridge).

SKETCH OF A PROOF. Through duality for planar multigraphs, flows become tensions or potential differences. Proposition 1 is a consequence of the following result: a multigraph $G$ is $k$-colorable $(k \geqslant 2)$ if and only if there is a potential difference in $G$ with values in $[-k+1,-1] \cup[1, k-1]$, this result is essentially Minty's Theorem on colorings of multigraphs [3] interpreted with the help of the "Feasible Potential Difference Theorem".

An easy and well-known consequence of Proposition 1 is that a planar multigraph is face colorable with 2 colors if and only if it is eulerian.

We are then led to the following

Definition. Let $H$ be a multigraph. A $[p, q]$-orientation of $H$ is an orientation $G$ of $H$ such that there is a flow in $G$ with values in $[p, q]$; if such an orientation $G$ exists, $H$ will be said to be $[p, q]$-orientable.

Proposition 1 now becomes

Proposition 2. A planar multigraph $H$ is face colorable with $k$ colors $(k \geqslant 2)$ if and only if it is $[1, k-1]$-orientable.

This is our motivation for the study of the $[p, q]$-orientability property for general multigraphs; as we shall see in the next section, this property is of interest even in the nonplanar case.

\section{II. [1,3]-orientability of cubic multigraphs and their chromatic index.}

THEOREM 1. Let $H$ be a cubic multigraph. $H$ is [1,3]-orientable if and only if it has chromatic index 3.

Proof. Suppose first that the edges of $H$ are colored with 3 colors $\alpha, \beta$ and $\gamma$. Let $G$ be any orientation of $H$, with every arc colored as the corresponding edge of $H$. It is easy to construct two flows $\phi^{\prime}$ and $\phi^{\prime \prime}$ in $G$ such that

arcs colored with $\alpha$ or $\beta$ have flow values +1 or -1 in $\phi^{\prime}$, arcs colored $\gamma$ have flow values 0 in $\phi^{\prime}$.

arcs colored with $\alpha$ or $\gamma$ have flow values +1 or -1 in $\phi^{\prime \prime}$, arcs colored $\beta$ have flow values 0 in $\phi^{\prime \prime}$.

Then $\phi^{\prime}+2 \phi^{\prime \prime}$ is a flow in $G$ with values in $[-3,-1] \cup[1,3]$; hence $H$ is $[1,3]$-orientable. 
Conversely let $G$ be an [1,3]-orientation of $H$ and $\phi$ a flow in $G$ with values in $[1,3]$. The value of an edge of $H$ will be defined as the value of $\phi$ in the corresponding arc of $G$. It will be easily seen that, due to the conservation of flow at each vertex, the edges of $H$ with value 2 form a perfect matching of $H$.

Hence the edges of $H$ with values 1 or 3 form a 2-factor $F$ of $H$; the orientation $G$ of $H$ defines an orientation $K$ of $F$. It will be easily checked that if we reverse each arc of $K$ corresponding to an edge of value 3 we obtain an orientation $K^{\prime}$ of $F$ such that:

$$
\forall v \in V(H)=V\left(K^{\prime}\right) \quad\left|d_{K^{\prime}}^{+}(v)-d_{K^{\prime}}^{-}(v)\right|=2 .
$$

Hence $F$ is bipartite and $H$ has chromatic index 3 . This completes the proof of Theorem 1.

Remark. According to Proposition 2, Theorem 1 is a generalization of the following result of Heawood [4]: A cubic planar 2-edge connected multigraph is face colorable with four colors if and only if it has chromatic index 3 .

\section{Balanced valuations of a multigraph.}

1. Definitions. Let $H$ be a multigraph. A balanced valuation of $H$ is a mapping $w$ of $V(H)$ into $R$ such that $\forall S \subseteq V(H)\left|\Sigma_{v \in s^{w}}(v)\right| \leqslant\left|\omega_{H}(S)\right|$. Bondy [5] has shown that a cubic graph $H$ has chromatic index 3 if and only if there is a balanced valuation of $H$ with values in $\{-2,+2\}$. We shall see that this result is a consequence of Theorem 1 .

2. Realization of a sequence of outdegrees by an orientation of a multigraph.

Proposition 3. Let $H$ be a multigraph with $V(H)=\left\{v_{1}, \ldots, v_{n}\right\}$. Let $k_{1}, \ldots, k_{n}$ be nonnegative integers. There is an orientation $G$ of $H$ such that $d_{G}^{+}\left(v_{i}\right)=k_{i} \forall i \in[1, n]$ if and only if the valuation $w$ of $H$ defined by

$$
\forall i \in[1, n] \quad w\left(v_{i}\right)=2 k_{i}-d_{H}\left(v_{i}\right)
$$

is balanced.

Proof. $w$ is balanced if and only if

$$
\forall S \subseteq V(H) \quad-\left|\omega_{H}(S)\right| \leqslant 2 \sum_{v_{i} \in S} k_{i}-\sum_{v_{i} \in S} d_{H}\left(v_{i}\right) \leqslant\left|\omega_{H}(S)\right|
$$

For every $S \subseteq V(H)$ let $H_{S}$ be the submultigraph of $H$ induced by $S$; then

$$
\sum_{v_{i} \in S} d_{H}\left(v_{i}\right)=2\left|E\left(H_{S}\right)\right|+\left|\omega_{H}(S)\right|
$$

Hence $w$ is balanced if and only if

$$
\forall S \subseteq V(H) \quad\left|E\left(H_{S}\right)\right| \leqslant \sum_{v_{i} \in S} k_{i} \leqslant\left|E\left(H_{S}\right)\right|+\left|\omega_{H}(S)\right|
$$

It will be easily checked that this condition is equivalent to the following

$$
\left\{\begin{array}{l}
\text { (i) } \forall S \subseteq V(H) \Sigma_{v_{i} \in S} k_{i} \geqslant\left|E\left(H_{S}\right)\right|, \\
\text { (ii) } \Sigma_{i \in[1, n]} k_{i}=|E(H)| .
\end{array}\right.
$$

Hakimi [6] has shown that conditions (i) and (ii) are necessary and sufficient for $H$ to have an orientation $G$ with $d_{G}^{+}\left(v_{i}\right)=k_{i} \forall i \in[1, n]$. This completes the proof. 
3. Nonnull flows and balanced valuations of a multigraph.

THEOREM 2. A multigraph $H$ is $[p, q]$-orientable $(0<p<q)$ if and only if there is a balanced valuation $w$ of $H$ such that

$\forall v \in V(H) \quad \exists k_{v} \in Z, \quad k_{v} \equiv d_{H}(v)(\bmod 2): w(v)=(q+p) k_{v} /(q-p)$.

Proof. Let $G$ be an orientation of $H$. According to the Feasible Flow Theorem $G$ is a $[p, q]$-orientation of $H$ if and only if

$$
\forall S \subseteq V(G)\left\{\begin{array}{l}
q\left|\omega_{G}^{+}(S)\right| \geqslant p\left|\omega_{G}^{-}(S)\right| \\
q\left|\omega_{G}^{-}(S)\right| \geqslant p\left|\omega_{G}^{+}(S)\right|
\end{array}\right.
$$

(The second condition is the first condition where $S$ is replaced by $V(G)-$ $S$.) This is equivalent to

$\forall S \subseteq V(G)\left\{\begin{array}{l}(q+p)\left(\left|\omega_{G}^{-}(S)\right|-\left|\omega_{G}^{+}(S)\right|\right) \leqslant(q-p)\left(\left|\omega_{G}^{-}(S)\right|+\left|\omega_{G}^{+}(S)\right|\right), \\ (q+p)\left(\left|\omega_{G}^{+}(S)\right|-\left|\omega_{G}^{-}(S)\right|\right) \leqslant(q-p)\left(\left|\omega_{G}^{+}(S)\right|+\left|\omega_{G}^{-}(S)\right|\right),\end{array}\right.$ or

$$
\frac{q+p}{q-p}|| \omega_{G}^{+}(S)|-| \omega_{G}^{-}(S)|| \leqslant\left|\omega_{G}(S)\right|=\left|\omega_{H}(S)\right| \quad \forall S \subseteq V(G) .
$$

But

$$
\begin{aligned}
\forall S \subseteq V(G)\left|\omega_{G}^{+}(S)\right|-\left|\omega_{G}^{-}(S)\right| & =\sum_{v \in S} d_{G}^{+}(v)-\sum_{v \in S} d_{G}^{-}(v) \\
& =\sum_{v \in S}\left(2 d_{G}^{+}(v)-d_{H}(v)\right) .
\end{aligned}
$$

Hence $G$ is a $[p, q]$-orientation of $H$ if and only if the valuation $w$ of $H$ defined by

$$
\forall v \in V(H) \quad w(v)=\frac{q+p}{q-p}\left(2 d_{G}^{+}(v)-d_{H}(v)\right)
$$

is balanced. The result now follows from Proposition 3 and from the fact that $(q+p) /(q-p)>1$.

REMARK. The values of any balanced valuation $w$ of a multigraph $H$ are restricted by the condition: $\forall v \in V(H)|w(v)| \leqslant d_{H}(v)$.

IV. Applications.

1. Cubic multigraphs.

Proposition 4. A cubic multigraph $H$ is $[p, q]$-orientable $(0<p<q)$ if and only if there is a balanced valuation of $H$ with values in

$$
\{-(q+p) /(q-p),(q+p) /(q-p)\}
$$

Proof. If $w(v)=(q+p) k_{v} /(q-p)$ with $|w(v)| \leqslant 3$ and $k_{v} \equiv 3(\bmod 2)$, then $\left|k_{v}\right|=1$; the result now follows from Theorem 2 .

Proposition 5 (Bondy [5]). A cubic multigraph has chromatic index 3 if and only if it has a balanced valuation with values in $\{-2,+2\}$.

This is a consequence of Theorem 1 and Proposition 4. 
Proposition 6. Let $w$ be a balanced valuation of a cubic multigraph $H$ with values in $\{-(q+p) /(q-p),(q+p) /(q-p)\}$; let

$$
W^{+}=\{v \in V(H) \mid w(v)>0\}
$$

and $W^{-}=V(H)-W^{+}$. Then $\left|W^{+}\right|=\left|W^{-}\right|$; moreover $H_{W^{+}}$and $H_{W^{-}}$are forests, each component of which having at most $[q / p]-1$ vertices $([q / p]$ is the integer part of $q / p$ ).

We omit the easy proof.

Proposition 7. A cubic multigraph is [1,2]-orientable if and only if it is bipartite.

Proof. According to Proposition 6, for $p=1$ and $q=2,\left(W^{+}, W^{-}\right)$form a coloring of $H$. Conversely, every bicoloring is of this type.

REMARK. Proposition 7 is a generalization of the well-known result [2] that a planar cubic multigraph is face colorable with 3 colors if and only if it is bipartite.

Proposition 8. Every planar cubic 2-edge connected multigraph has a balanced valuation with values in $\{-5 / 3,5 / 3\}$.

This is exactly the Five Color Theorem for planar multigraphs, formulated with the help of Propositions 2 and 4.

PROPOSITION 9. The vertices of every planar cubic 2-edge connected multigraph can be colored with 2 colors in such a way that

The two colors form two sets of vertices of the same cardinality.

There is no monochromatic elementary cycle and no monochromatic tree on 4 vertices.

This is a direct consequence of Propositions 8 and 6 .

2. Other applications. Any result or problem about colorings of planar graphs could be stated in a "balanced valuation" formulation. For instance:

Four Color Problem. Does every 2-edge connected planar multigraph $H$ have a balanced valuation $w$ with $w(v) \equiv 2 d_{H}(v)(\bmod 4) \forall v \in V(H)$ ?

Remark. The Four Color Problem can be restricted to cubic multigraphs, or to 5-regular multigraphs; in both cases the corresponding balanced valuations have values in $\{-2,+2\}$.

Proposition 10. A planar 2-edge connected multigraph $H$ is face colorable with 3 colors if and only if it has a balanced valuation $w$ with $w(v) \equiv 3 d_{H}(v)$ $(\bmod 6) \forall v \in V(H)$.

Remarks. From a theorem of Grötzsch [7] this is true for 4-edge connected planar multigraphs.

For 5-regular or 7-regular multigraphs the corresponding balanced valuations have values in $\{-3,+3\}$.

Propositions 10 and 3 allow us to write:

Proposition 11. A planar 2-edge connected multigraph $H$ is face colorable with 3 colors if and only if there is an orientation $G$ of $H$ such that:

$$
\forall v \in V(G) d_{G}^{+}(v)-d_{G}^{-}(v) \equiv 0(\bmod 3) .
$$


It can be seen that Heawood's result [4] relating vertex assignments modulo 3 and edge colorations with 3 colors in a planar cubic multigraph $H$ is a consequence of Proposition 11 applied to the "radial graph" of $H$.

3. Further problems. So far we have been concerned only with $[p, q]$ orientability for $p=1$. An infinite class of problems arises when we consider other values of $p$. For instance:

CONJECTURE. Every planar 2-edge connected multigraph is [2,7]-orientable. This would be better than the Five Color Theorem but weaker than the Four Color Theorem.

Notes ADDED IN PROOF (SePTEMBer 1975). (1) It can be proved, using a result of Tutte (Theorem 5.44 in [8]), that a multigraph $H$ is $[1, k-1]$ orientable $(k \geqslant 2)$ if and only if every orientation $G$ of $H$ has a colour-cycle (nowhere-zero flow) over $Z_{k}$ (the cyclic group of order $k$ ); see [9] and [10].

Hence [1, $k-1]$-orientability can be studied using Tutte's theory of dichromatic polynomials [9].

(2) Theorem 1 has been proved by Minty in [11]; an elementary proof appears in [10].

(3) Tutte has conjectured that:

every 2-edge-connected multigraph is [1, 4]-orientable (see [9]);

every 4 -edge-connected multigraph is $[1,2]$-orientable.

It is proved in [10] that:

every 2-edge-connected multigraph is [1,7]-orientable;

every 4-edge-connected multigraph is $[1,3]$-orientable.

\section{REFERENCES}

1. C. Berge, Graphes et hypergraphes, Dunod, Paris, 1974.

2. O. Ore, The four-color problem, Pure and Appl. Math., vol. 27, Academic Press, New York and London, 1967. MR 36 \#4.

3. G. J. Minty, A theorem on n-coloring the points of a linear graph, Amer. Math. Monthly 69 (1962), 623-624.

4. P. J. Heawood, On the four-colour map theorem, Quart. J. Math. 19(1898), 270-285.

5. J. A. Bondy, Balanced colourings and the four colour conjecture, Proc. Amer. Math. Soc. 33(1972), 241-244. MR 45 \#3246.

6. S. L. Hakimi, On the degrees of the vertices of a directed graph, J. Franklin Inst. 279(1965), 290-308. MR31 \#4736.

7. H. Grötzsch, Zur Theorie der diskreten Gebilde, VII. Ein Dreifarbensatz für dreikreisfreie Netze auf der Kugel, Wiss. Z. Martin-Luther-Univ. Halle-Wittenberg. Math.-Nat. Reihe 8(1958/ 59), 109-120. MR22 \#7113c.

8. W. T. Tutte, Lectures on matroids, J. Res. Nat. Bur. Standards Sect. B 69B (1965), 1-47. MR 31 \#4023.

9. A contribution to the theory of chromatic polynomials, Canad. J. Math. 6 (1954), 80-91. MR 15, 814.

10. F. Jaeger, On nowhere zero flows in multigraphs, Proc. Fifth British Combinatorial Conference, Aberdeen, July 1975 (to appear).

11. G. J. Minty, A theorem on three-coloring the edges of a trivalent graph, J. Combinatorial Theory 2 (1967), 164-167. MR 34 \#5703.

I.R.M.A., Université Scientifique et Médicale de Grenoble, Boite Postale 53, 38041 Grenoble/Cédex, France 